\title{
2. Nationaler Grippeimpftag der Schweizer Hausärztinnen/-ärzte am 4. November 2005
}

\author{
Kollegium für Hausarztmedizin in Zusammenarbeit mit SGAM, SGIM, SGP und FMP, unterstützt von BAG und FMH
}

\section{U. Grüninger}

Korrespondenz: Dr. med. Ueli Grüninger Landhausweg 26 CH-3007 Bern Tel. 0313700670 Fax 0313700679
Der Grippeimpftag ist eine Gesundheitsinitiative der Organisationen der Schweizer Hausärztinnen und Hausärzte: KHM (Federführung), SGAM, SGIM und SGP, unterstützt von BAG und FMH [1].

Die Schweizer Hausärztinnen und Hausärzte bieten all jenen Risikopersonen, die sich bis anhin noch nicht gegen Grippe impfen liessen,

- einen leichten Zugang zur Grippeimpfung in unseren Hausarztpraxen;

- ohne Anmeldung und mit minimalen Formalitäten;

- zu einem Sonderpreis von Fr. 25.-.

Ausführliche Informationen sowie Hilfsmittel für die Durchführung des Grippeimpftags in der Praxis können von der Grippeimpfseite der KHM-Homepage www.kollegium.ch herabgeladen werden.

Als Dienstleistung für Impfinteressierte veröffentlicht das Kollegium für Hausarztmedizin auf seiner Homepage ferner regionale Adresslisten der am Grippeimpftag teilnehmenden Arztpraxen. Wir bitten deshalb die Kolleginnen und Kollegen, in ihrer Region wenn immer möglich eine Liste der Impfpraxen zu erstellen und diese einerseits der lokalen Presse, andererseits dem Kollegium für Hausarztmedizin (zur Publikation auf der Homepage des KHM, E-Mail: ursula.laubscher@kollegium.ch) zur Verfügung zu stellen.

\section{Kontaktstellen und Adressen für mehr Infos}

- KHM: www.kollegium.ch; Dr. med. Ueli Grüninger, Ursula Laubscher, Geschäftsstelle, Landhausweg 26, 3007 Bern, Tel. 031 37006 70/71/72, Fax 03137006 79, E-Mail: ursula.laubscher@kollegium.ch.

- BAG: www.grippe.admin.ch; für Bestellungen von Informationsmaterial: Dokumentationsstelle Grippeprävention, Postfach, 3000 Bern 6, Tel. 03135214 60, Fax 03135214 71, E-Mail: grippe@bag.admin.ch, Internet:www. bag.admin.ch/grippe/kampagne/d/bestell. htm.

\section{Literatur}

1 Grüninger U. Nationaler Grippeimpftag der Schweizer HausärztInnen: am 4. November 2005 zum zweiten Mal. PrimaryCare 2005;5:388-9. 